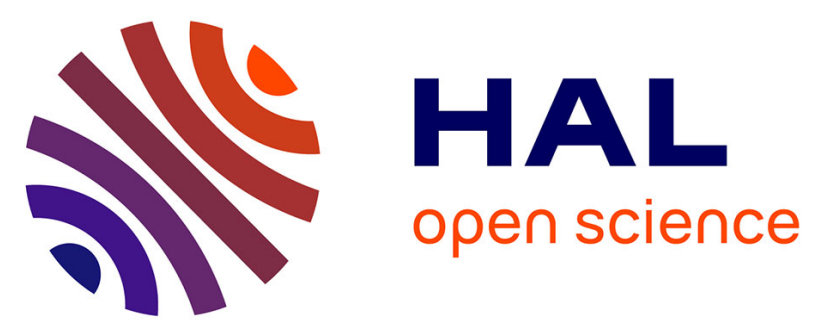

\title{
Aging Effect and Induced Electric Phenomena on Dielectric Materials Irradiated With High Energy Electrons
}

Thierry Paulmier, Rachelle Hanna, Mohamed Belhaj, Bernard Dirassen, Denis Payan, Nicolas Balcon, Claire Tonon, Eric Dantras, Alain Bernès

\section{To cite this version:}

Thierry Paulmier, Rachelle Hanna, Mohamed Belhaj, Bernard Dirassen, Denis Payan, et al.. Aging Effect and Induced Electric Phenomena on Dielectric Materials Irradiated With High Energy Electrons. IEEE Transactions on Plasma Science, 2013, vol. 41 ( $\mathrm{n}^{\circ}$ 12), pp. 3422-3428. 10.1109/TPS.2013.2279302 . hal-01166254

\section{HAL Id: hal-01166254 \\ https://hal.science/hal-01166254}

Submitted on 22 Jun 2015

HAL is a multi-disciplinary open access archive for the deposit and dissemination of scientific research documents, whether they are published or not. The documents may come from teaching and research institutions in France or abroad, or from public or private research centers.
L'archive ouverte pluridisciplinaire HAL, est destinée au dépôt et à la diffusion de documents scientifiques de niveau recherche, publiés ou non, émanant des établissements d'enseignement et de recherche français ou étrangers, des laboratoires publics ou privés. 


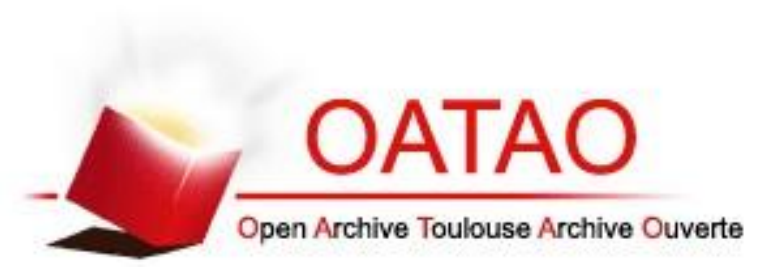

Open Archive Toulouse Archive Ouverte (OATAO)

OATAO is an open access repository that collects the work of Toulouse researchers and makes it freely available over the web where possible.

This is an author-deposited version published in: http://oatao.univ-toulouse.fr/ Eprints ID: 13975

Identification number: DOI: 10.1109/TPS.2013.2279302

Official URL: http://dx.doi.org/10.1109/TPS.2013.2279302

\section{To cite this version:}

Paulmier, Thierry and Hanna, Rachelle and Belhaj, Mohamed and Dirassen, Bernard and Payan, Denis and Balcon, Nicolas and Tonon, Claire and Dantras, Eric and Bernès, Alain Aging Effect and Induced Electric Phenomena on Dielectric Materials Irradiated With High Energy Electrons. (2013) IEEE Transactions on Plasma Science, vol. 41 ( $\mathrm{n}^{\circ}$ 12). pp. 3422-3428. ISSN 00933813

Any correspondence concerning this service should be sent to the repository administrator: staff-oatao@inp-toulouse.fr 


\title{
Aging Effect and Induced Electric Phenomena on Dielectric Materials Irradiated With High Energy Electrons
}

\author{
Thierry Paulmier, Rachelle Hanna, Mohamed Belhaj, Bernard Dirassen, Denis Payan, \\ Nicolas Balcon, Claire Tonon, Eric Dantras, and Alain Bernès
}

\begin{abstract}
The high radiation dose received by space used polymers may greatly alter their electric properties. This effect could, for instance, reduce significantly radiation-induced conductivity (RIC) leading to high charging risks that were not predicted from pristine sample. For an optimized qualification and prediction, it is therefore highly important to characterize the charging properties of polymers and their evolution as a function of the received dose. This paper aimed at studying aging of electric properties of four different polymers (Teflon FEP, Kapton, polyepoxy DP 490 adhesive, and silicon QS1123 adhesive) at high dose level $\left(10^{5}\right.$ and $\left.10^{6} \mathrm{~Gy}\right)$. We have been able to demonstrate that aging could lead to the reduction of RIC on some polymers (for polyepoxy and silicone adhesives, and FEP) or inversely to the increase of RIC on others (e.g., Kapton). Ionization effect must, however, be considered in the analysis of the results. Relaxation processes could drastically affect the charging profile and macroscopic electric properties.
\end{abstract}

Index Terms-Aging, dielectric materials, electron radiation effects, materials reliability, space technology.

\section{INTRODUCTION}

D IELECTRIC materials used in space on satellite external surfaces may have to cope with strong levels of radiation dose under proton and electron irradiations. These high radiation dose levels received after long flight duration (several years flight) may strongly affect the material structure through fragmentation and reticulation processes, defects production, or ionization. Polymer materials are especially highly sensitive

T. Paulmier, M. Belhaj, and B. Dirassen are with Office National d'Etudes et de Recherches Aérospatiales, The French Aerospace Laboratory, Toulouse F-31055, France (e-mail: thierry.paulmier@onera.fr; mohamed.belhaj@ onera.fr; bernard.dirassen@onera.fr).

R. Hanna is with Office National d'Etudes et de Recherches Aérospatiales, The French Aerospace Laboratory, Toulouse F-31055, France, and also with the Centre National d'Etudes Spatiales, Toulouse Cedex 9 31401, France (e-mail: rachelle.hanna@hotmail.com).

D. Payan and N. Balcon are with the Centre National d'Etudes Spatiales, Toulouse Cedex 9 31401, France (e-mail: denis.payan@cnes.fr; nicolas.balcon@cnes.fr).

C. Tonon is with Astrium Satellites, Toulouse Cedex 4 31402, France (e-mail: claire.tonon@astrium.eads.net).

E. Dantras and A. Bernès are with Centre Interuniversitaire de Recherche et d' Ingénierie des Matériaux, Université Paul Sabatier, Toulouse Cedex 9 31062, France (e-mail: eric.dantras@univ-tlse3.fr; alain.bernes@univ-tlse3.fr).

Digital Object Identifier 10.1109/TPS.2013.2279302 to ionizing electron radiation and their physicochemical or electronic structure may be modified during electron irradiation. Their electric behavior, such as radiation-induced conductivity (RIC), under geostationary electron flux can then be noticeably altered through high radiation dose effect. This aging effect can be quite different from one material to the other and is therefore a new and important factor to consider during risk management. Former studies have indeed demonstrated through injection of high radiation dose [1] and successive irradiation steps [2] that charging behavior of space used polymers is strongly affected by radiation history of the material. Teflon FEP material then gets highly sensitive with the increasing radiation dose, whereas reverse behavior is observed on Kapton polyimide materials that tends to get highly conductive.

For this paper, aging of electric properties through high energy electron irradiation has been characterized with ground experimental tests: this paper was focused on aging of $100-\mu$ m-thick polymer films (Teflon FEP, Kapton) and adhesives (polyepoxy Scotch-Weld DP490 and silicone MAP QS1123). These materials have been submitted to two radiation doses: $10^{5} \mathrm{~Gy}$ (for Teflon and Kapton) and $10^{6} \mathrm{~Gy}$ (for all materials). RIC has been measured on both pristine and aged samples. Long term ionization effect on the electric properties of polymer materials has also been characterized through successive irradiation phases and adjustment on the relation phase duration: this task is of high importance to discriminate between aging effect because of physicochemical evolution in the material and influence of radiation dose through ionization process.

\section{Experimental SetuP AND Protocol}

\section{A. Experimental Setup}

The different experimental tests that will be described in Section II-B have been performed in the SIRENE facility installed at ONERA (Toulouse, France). This facility has been funded by CNES and is operated at ONERA. SIRENE (Fig. 1) is a sophisticated and unique test facility especially designed for the study of surface and internal charging of space materials and satellite components under extreme environment (usually geostationary orbit radiation conditions). This facility is able to reproduce the geostationary electron spectrum (in the energy range [0-400 keV]). It allows realistic assessment of voltage built up in geostationary orbit. Its flexibility allows as 


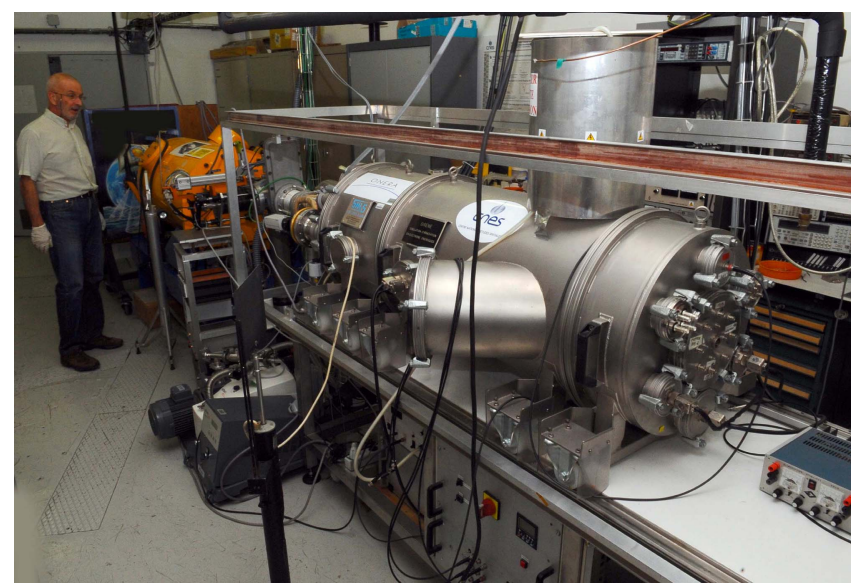

Fig. 1. SIRENE experimental facility.

well simulation of extreme environments that can be different than the geostationary one (e.g., MEO, LEO or other planetary radiation environments). This facility is also widely used for the characterization of radiation-induced conductivities of space materials in vacuum because of the use of a penetrating 400-keV electron beam that stimulates the potential decay during the relaxation phase without inducing any bulk charging. Using this high energy electron beam and a high incoming flux on the sample (up to $5 \mathrm{nA} \cdot \mathrm{cm}^{-2}$ ), we are able as well to perform radiation aging on the material. Space materials, such as polymers (Kapton, Teflon, etc.), adhesives or coverglasses can then be aged in SIRENE with equivalent flight durations equal to several months or years. The experimental protocol applied for RIC evaluation and aging process is described in detail in the following section.

The electron spectrum simulation is achieved using two monoenergetic electron beams ( 20 and $400 \mathrm{keV}$ ). These two beams are diffused in energy and angle to produce a spacelike electron flow with a good flux homogeneity in a diameter equal to $20 \mathrm{~cm}$ on the sample holder. The use of these two energy values is chosen to get a good fit in regard of the GEO Kp $>5$ electron spectrum, as shown in Fig. 2. A complex diffusion foil is used to diffuse in energy the incident $400-\mathrm{keV}$ electron beam and get the GEO-like distributed energy spectrum. The low energy $20-\mathrm{keV}$ electron beam is used for simulation of critical charging conditions while the $400-\mathrm{keV}$ distributed part acts especially on the initiation of bulk radiation-induced conductivity that tends to lower significantly charging levels in dielectric materials. The nominal integrated fluxes used for the $20-\mathrm{keV}$ monoenergetic beam and distributed $0-400 \mathrm{keV}$ one are, respectively, equal to 250 and 50 pA.cm ${ }^{-2}$ but these different fluxes can be changed independently. The temperature of the sample holder can be controlled in the range $\left[-180{ }^{\circ} \mathrm{C}, 150{ }^{\circ} \mathrm{C}\right]$ allowing reproducing the temperature variations of materials on flight. A pumping system allows performing experiments at vacuum of $10^{-6} \mathrm{hPa}$.

\section{B. Experimental Protocol}

The experimental protocol has been divided in two phases.

1) Phase 1: The first phase deals with the characterization of electrical aging of polymers (Teflon, Kapton, polyepoxy

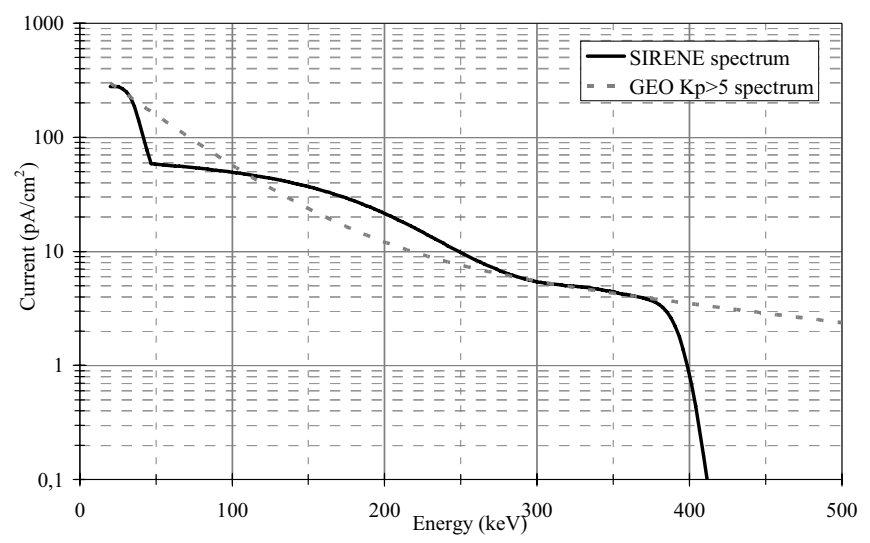

Fig. 2. SIRENE electron beam spectral characteristics and GEO Kp $>5$ integrated reference spectrum.

adhesives Scotch Weld DP490, and silicon adhesives MAP QS1123). RIC has been assessed on pristine and aged samples. The samples have been aged using a monoenergetic 400-keV electron beam that get through the sample and inject a high radiation dose in the bulk. The tested samples are $100-\mu \mathrm{m}$ thick. The dose distribution in the bulk then keeps up the same value. Teflon and Kapton have been tested at two different dose levels: 1) $10^{5}$ Gy and 2) $10^{6}$ Gy. Polyepoxy DP490 and silicon QS1123 have been aged at a dose level of $10^{6} \mathrm{~Gy}$. The aging radiative process has been performed with fluxes equal to $2.5 \mathrm{nA} . \mathrm{cm}^{-2}$ for $10^{5}$ Gy dose level (irradiation duration: $5 \mathrm{~h}$ ) and $5 \mathrm{nA} . \mathrm{cm}^{-2}$ for $10^{6} \mathrm{~Gy}$ dose level (irradiation duration: $30 \mathrm{~h}$ ). For the evaluation of electric conductivity, the potential decay method was applied: for this method, bulk induced conductivity is assessed through the analysis of the electric voltage relaxation after charging the sample with low energy electrons $(20 \mathrm{keV})$ up to a given surface potential [1]. During the electric voltage relaxation, a 400-keV penetrating ionizing electron beam (which does not contribute to material charging) is used to stimulate the voltage decay through the activation of the radiation-induced conductivity. Fig. 3 shows this test method for RIC evaluation. The model of a capacitor relaxing its charge through a resistance is then applied. The time constant of the discharge (RC) is determined and ultimately the conductivity $\sigma$

$$
\sigma=\varepsilon \frac{d V_{s} / d t}{V_{s}}
$$

for which $\sigma$ is the bulk electric conductivity, $\varepsilon$ is the relative permittivity of the material, and $\mathrm{V}_{s}$ is the surface potential of the material.

Instantaneous dose effects are quite important and can induce radiation conductivities higher than the intrinsic bulk conductivities by several orders of magnitude. RIC depends on radiation dose rate $d D / d t$ and two parameters $k$ and $\Delta$ characteristic of each material

$$
\mathrm{RIC}=k\left(\frac{d D}{d t}\right)^{\Delta}
$$

where $D$ is the dose, $d D / d t$ is the dose rate, $\Delta$ is a coefficient (without unit) $<1$, and $k$ is the RIC coefficient. 

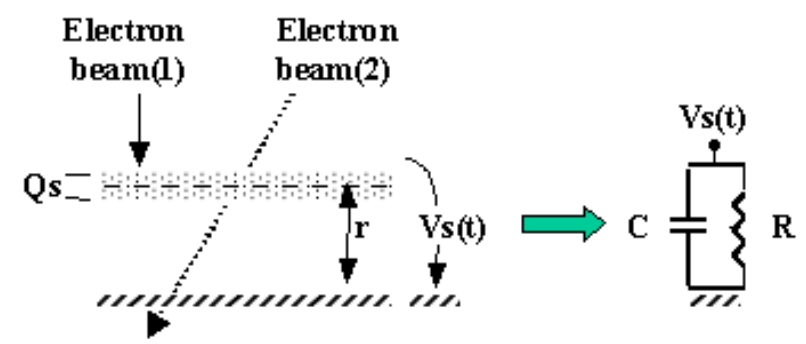

Fig. 3. Surface voltage decay method for RIC evaluation. Electron beam 1: charging beam and electron beam. Electron beam 2: decay stimulating beam for RICassessment.

TABLE I

EXPERIMENTAL PROTOCOL FOR RIC EXTRACTION ON Pristine ANd $10^{5}$ Gy Aged SAMples

\begin{tabular}{|c|c|c|c|c|}
\hline & $\begin{array}{l}20 \mathrm{keV} \text { beam } \\
\text { Flux }\left(\mathrm{pA} \mathrm{cm}^{-2}\right)\end{array}$ & \multicolumn{2}{|c|}{$400 \mathrm{keV}$ beam } & \multirow[t]{2}{*}{ Duration } \\
\hline & & $\begin{array}{l}\text { Radiation dose rate } \\
\left(\mathrm{mGy} . \mathrm{s}^{-1}\right)\end{array}$ & $\begin{array}{c}\text { Flux } \\
\left(\mathrm{pA} \cdot \mathrm{cm}^{-2}\right)\end{array}$ & \\
\hline Sample charging & 100 & 0 & 0 & $20 \mathrm{~min}$. \\
\hline Intrinsic relaxation & 0 & 0 & 0 & $5-10 \mathrm{~min}$. \\
\hline \multirow{3}{*}{$\begin{array}{l}\text { Stimulated } \\
\text { relaxation }\end{array}$} & \multirow{3}{*}{0} & 4 & 9 & $\begin{array}{l}\text { up to null } \\
\text { potential }\end{array}$ \\
\hline & & 20 & 48 & $\begin{array}{l}\text { up to null } \\
\text { potential }\end{array}$ \\
\hline & & 100 & 240 & $\begin{array}{l}\text { up to null } \\
\text { potential }\end{array}$ \\
\hline
\end{tabular}

TABLE II

EXPERIMENTAL PROTOCOL FOR RIC EXTRACTION ON PRistine AND $10^{6}$ Gy Aged SAMPLeS

\begin{tabular}{|c|c|c|c|c|}
\hline & $\begin{array}{l}20 \mathrm{keV} \text { beam } \\
\text { Flux }\left(\mathrm{pA} . \mathrm{cm}^{-2}\right)\end{array}$ & \multicolumn{2}{|c|}{$400 \mathrm{keV}$ beam } & \multirow[t]{2}{*}{ Duration } \\
\hline & & $\begin{array}{l}\text { Radiation dose rate } \\
\left(\mathrm{mGy} . \mathrm{s}^{-1}\right)\end{array}$ & $\begin{array}{c}\text { Flux } \\
\left(\mathrm{pA} \cdot \mathrm{cm}^{-2}\right)\end{array}$ & \\
\hline Sample charging & 100 & 0 & 0 & $20 \mathrm{~min}$. \\
\hline Intrinsic relaxation & 0 & 0 & 0 & $5-10 \mathrm{~min}$. \\
\hline \multirow{4}{*}{$\begin{array}{l}\text { Stimulated } \\
\text { relaxation }\end{array}$} & \multirow{4}{*}{0} & 0.5 & 1.2 & $\begin{array}{l}\text { up to null } \\
\text { potential }\end{array}$ \\
\hline & & 4 & 9 & $\begin{array}{l}\text { up to null } \\
\text { potential }\end{array}$ \\
\hline & & 20 & 48 & $\begin{array}{l}\text { up to null } \\
\text { potential }\end{array}$ \\
\hline & & 100 & 240 & $\begin{array}{l}\text { up to null } \\
\text { potential }\end{array}$ \\
\hline
\end{tabular}

Four different dose rates $(1.2,9,48$, and $240 \mathrm{mGy} / \mathrm{s})$ have been applied on the monoenergetic $400-\mathrm{keV}$ electron beam for RIC evaluation through stimulated relaxation analysis. The $k$ and $\Delta$ parameters can be extracted by representing $\operatorname{Ln}\left(\sigma_{\mathrm{RIC}}\right)$ as a function of $\operatorname{Ln}(d D / d t)$. Using (2), we can then write $L n \sigma=L n k+\Delta L n(d D / d t)$ and then derive $k$ and $\Delta$. This representation allows a validation of equation.

Before the initiation of RIC with the $400-\mathrm{keV}$ electron beam, intrinsic relaxation has been performed after the $20-\mathrm{keV}$ electron irradiation to provide a comparison between intrinsic and radiation-induced bulk conductivity. The duration applied on intrinsic relaxation has been adjusted between 5 and $15 \mathrm{~min}$ depending on time needed to reach good estimation for bulk intrinsic conductivity. Tables I and II list the experimental protocols applied for RIC evaluation on pristine and aged samples $\left(10^{5}\right.$ and $\left.10^{6} \mathrm{~Gy}\right)$.

2) Phase 2: The second phase deals with the influence of low radiation dose on the electric properties of Teflon FEP. Successive irradiations have been performed on this material
TABLE III

EXPERIMENTAL PROTOCOL FOR SUCCESSIVE IRRADIATION PROCESS ON TEFLON FEP

\begin{tabular}{|c|c|c|}
\cline { 2 - 3 } \multicolumn{1}{c|}{} & Process & Duration \\
\hline Step 1 & $\begin{array}{c}\text { Irradiation 1: } \\
\text { Step 2 }\end{array}$ & $180 \mathrm{~min}$. \\
\hline Step 3 & Relaxation & 3 hours or 1 week \\
\hline $\begin{array}{c}\text { Irradiation 2: } \\
\text { 2 }\end{array}$ & 180 min. \\
\hline
\end{tabular}

with charging and ionizing electron beams. The different experimental tests have been performed at room temperature. A $100-\mu$ m-thick Teflon FEP sample was then irradiated simultaneously by two electron beams $\left(20 \mathrm{keV}, 250\right.$ pA.cm ${ }^{-2}$ $+400 \mathrm{keV}, 50 \mathrm{pA} \cdot \mathrm{cm}^{-2}$ ). The sample is then stored under high vacuum, at $10^{-6} \mathrm{hPa}$, during a given period $(3 \mathrm{~h}$ or 1 week) and then irradiated again with the same electron spectrum. The relaxation duration has been varied to study ionization effect on the macroscopic electric properties of Teflon FEP. Evolution of surface potential as a function of irradiation time has been recorded during both irradiations steps. Both potential profiles have been compared on the same graph to get a better understanding of long term ionization effect upon radiation-induced conductivity of this material. This experiment is therefore composed of two tests.

1) Test $1:$ relaxation duration equal to 3 hours.

2) Test 2 : relaxation duration equal to one week.

For each test, a new sample has been used. Table III lists the experimental protocol applied on each test.

\section{EXPERIMENTAL RESULTS}

We present in the following sections the different experimental results of phase 1 and 2 .

\section{A. Teflon FEP}

Former studies [1] have demonstrated that RIC of Teflon FEP is significantly altered by electron irradiation at high dose levels $\left(10^{5} \mathrm{~Gy}\right)$. The following results highlight new features in regard of RIC dependence with the received radiation dose $\left(10^{5}\right.$ and $\left.10^{6} \mathrm{~Gy}\right)$. Fig. 4 shows the evolution of charging potential for pristine and $10^{5}$ Gy aged FEP sample along the different steps described in Section II-B for phase 1 (charging with $20 \mathrm{keV}$, intrinsic relaxation, and enhanced relaxation with 400-keV electron beam). We can first notice that charging kinetics are similar for pristine and aged samples meaning that aged FEP keeps up a very low intrinsic conductivity, close to $10^{-20} \Omega^{-1} \cdot \mathrm{m}^{-1}$. From the enhanced relaxation phases, we can notice that, for the three tested radiation dose rates, the aged sample presents a lower relaxation kinetics that demonstrates a lower RIC for the aged FEP at this radiation dose level. This feature is conspicuous in Fig. 5 showing the evolution of RIC as a function of radiation dose rate for both FEP samples (pristine and aged). We can clearly notice a reduction of RIC with the radiation dose at this level. The effect of radiation dose could therefore act in different ways: evolution of structure through chain scission and crosslinking effect or implantation of charges (electrons and holes) 


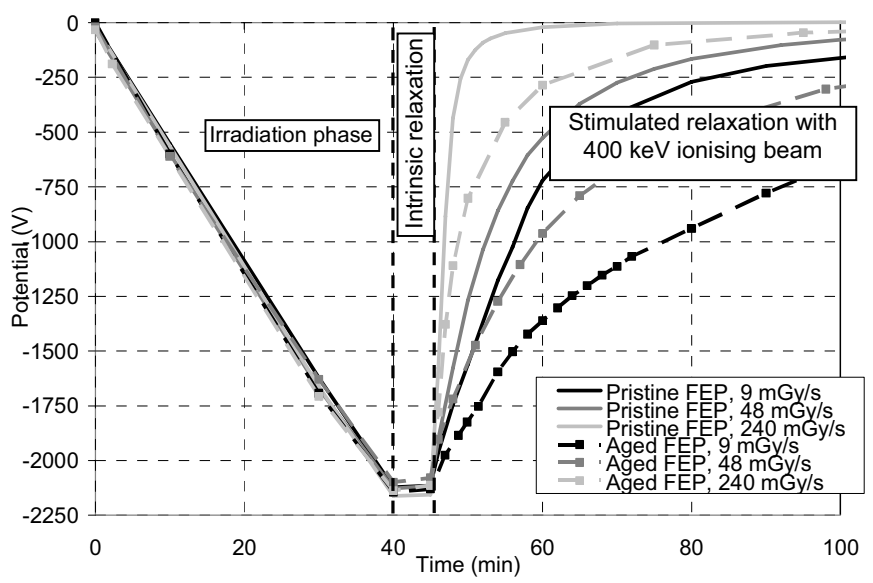

Fig. 4. Evolution of surface potential on pristine and aged FEP samples (radiation dose: $10^{5} \mathrm{~Gy}$ ) during the different experimental steps for intrinsic and RIC evaluation. Step 1: irradiation with $20 \mathrm{keV}$ electron beam. Step 2: intrinsic relaxation. Step 3: stimulated relaxation with $400-\mathrm{keV}$ electron beam.

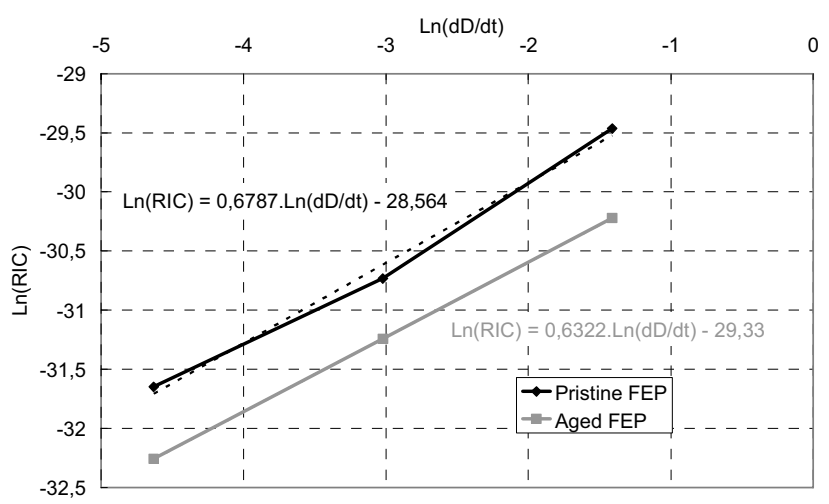

Fig. 5. Evolution of RIC as a function of radiation dose rate for pristine and aged samples (radiation dose: $10^{5} \mathrm{~Gy}$ ) for Teflon FEP.

in deep traps. The first process might induce the formation of new charge traps and consequently an alteration of the trap energy distribution. This process should therefore modify the RIC profile and especially the $\Delta$ factor that is an image of charge trap distribution in the band diagram [3]. Electron trapping because of radiation dose could induce, as we will see further in this paper, an increase of recombination centers for holes (free holes can recombine with trapped electrons) [4], a consequent reduction of free holes in the valence band and reduction of RIC. This sole trapping effect would not affect the slope of RIC curve [ $\operatorname{Ln}(\mathrm{RIC})$ as a function of $\operatorname{Ln}(d D / d t)]$ that corresponds to parameter $\Delta$. RIC is indeed directly dependent on free holes density. The reduction of free holes density because of electron trapping would therefore shift RIC to lower values, which affects basically parameter $k$ of equation. Parameter $\Delta$ in the same equation is only dependent on trap energy distribution of electrons and holes [3] and does not then vary with ionization level and trapping or recombination kinetics.

Fig. 5 shows that RIC curve slope is not strongly modified meaning that ionization effect through charge trapping steers at great level the observed RIC variation. A structural evolution of the material under high radiation dose might occur within

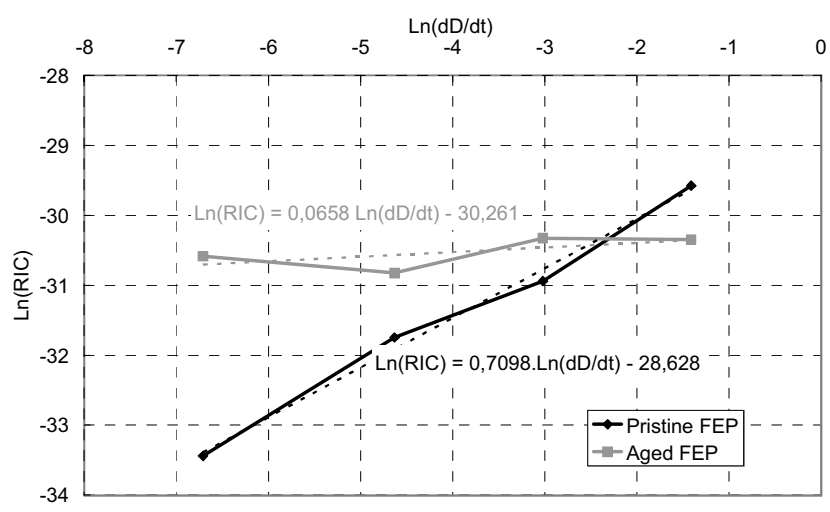

Fig. 6. Evolution of RIC as a function of radiation dose rate for pristine and aged samples (radiation dose: $10^{6} \mathrm{~Gy}$ ) for Teflon FEP.

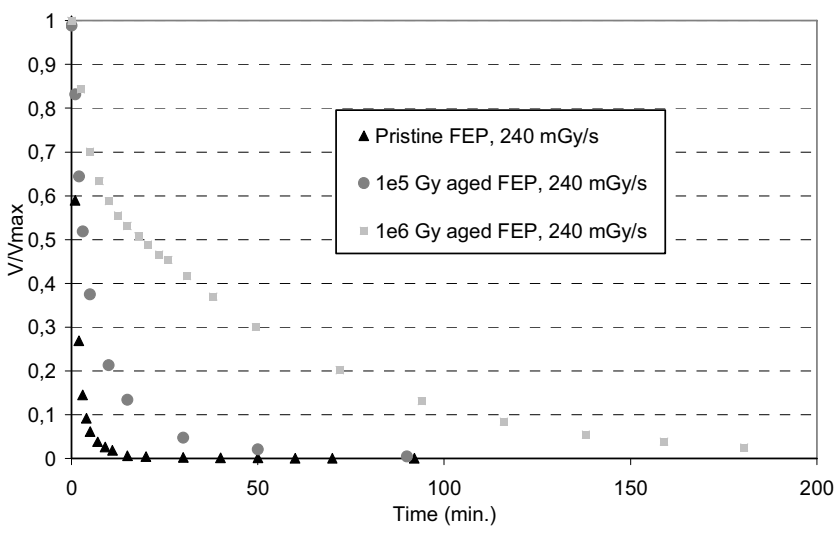

Fig. 7. Evolution of charging surface potential measured on both pristine and aged FEP samples as a function of relaxation time for a radiation dose rate equal to $240 \mathrm{mGy} / \mathrm{s}$.

the material but would not be the major effect ruling on the conductivity in FEP. The situation, as seen further, is quite different at higher dose.

Fig. 6 shows the evolution of RIC as a function of radiation dose rate for pristine FEP sample and the $10^{6}$ Gy aged FEP sample. It is interesting to notice a significant change in the slope of the aged curve, meaning that radiation dose has drastically modified the structure of the materials, and certainly the band structure as well as the traps distribution profile. The effect of radiation dose on RIC and charging kinetics is conspicuous on Fig. 7 showing the evolution of charging potential at $240 \mathrm{mGy} / \mathrm{s}$ for pristine FEP, $10^{5}$ Gy aged sample, and $10^{6} \mathrm{~Gy}$ aged sample. We can indeed notice that relaxation kinetics on aged samples at this radiation dose rate is lower than for pristine FEP sample for both radiation dose level but the effect is enhanced at $10^{6} \mathrm{~Gy}$.

The ionization process may as well affect at significant level the electrical properties of FEP sample, especially for low received radiation dose level. We have demonstrated (phase 2 of the experimental protocol described in Section II-B) that electrical properties of polymers can be altered by charge implantation in the material bulk. A FEP sample was irradiated simultaneously by two electron beams $(20 \mathrm{keV}, 250 \mathrm{pA} / \mathrm{cm} 2+$ $400 \mathrm{keV}, 50 \mathrm{pA} / \mathrm{cm} 2$ ) and after $3 \mathrm{~h}$ or 1 week relaxation (no irradiation), the sample was similarly reirradiated with the 


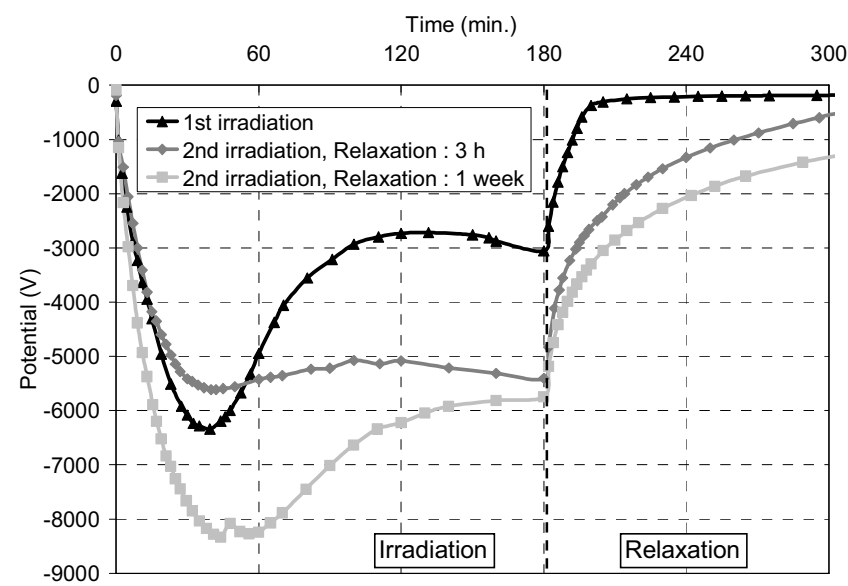

Fig. 8. Evolution of charging surface potential on FEP under successive irradiations with $\left(20 \mathrm{keV}, 250 \mathrm{pA} . \mathrm{cm}^{-2}+400 \mathrm{keV}, 50 \mathrm{pA} . \mathrm{cm}^{-2}\right)$ and variation of relaxation duration between both irradiations: $3 \mathrm{~h}$ or 1 week.

same electron beam configuration. The charging evolution for the first and second rounds of radiation is shown in Fig. 8.

We can clearly notice that both charging profile are similar but that the preirradiated sample tends to get more resistive. Adjustment of relaxation time between both irradiation steps revealed that this parameter strongly acts on the charging profile (and therefore on the apparent electrical properties) of the preirradiated sample (Fig. 8). For low relaxation duration, the preirradiated sample tends to get more conductive. This has been ascribed to the fact that concentration of free holes kept up a high level because of high trapping time, inducing then a delayed apparent conductivity when the second irradiation is initiated. For high relaxation time, density of free holes is low but trapped electrons (generated during the first ionization step) act as recombination centers that tend to reduce holes generation in the valence band and RIC, as mentioned previously.

Ionization effect should therefore be considered in the analysis of the experimental results and especially for devising the experimental protocol for parameters extraction.

\section{B. Kapton}

The second polymer that has been tested at high radiation dose is Kapton. Figs. 9 and 10 show the evolution of RIC as a function of radiation dose rate for pristine Kapton sample and Kapton samples aged at $10^{5}$ Gy and $10^{6} \mathrm{~Gy}$. We can clearly notice a strong evolution of RIC profile with the radiation dose level. At $10^{5} \mathrm{~Gy}$, the aged sample is noticeable more conductive than the pristine sample. For a dose rate of 1.2 $\mathrm{mGy} / \mathrm{s}$ and a surface potential of $-50 \mathrm{~V}$, the RIC is then equal to $3.10^{-15} \Omega^{-1} \cdot \mathrm{m}^{-1}$ for the pristine sample versus $4.3 \times 10^{-14}$ for the aged sample. At this radiation dose level, ionization may prevail over structural aging effect. Trapped electrons and holes are gradually detrapped in the conduction and valence bands, which tends to maintain a high density for free electrons and holes and therefore a high RIC. On the contrary, at $10^{6}$ Gy, the aged sample recover the electrical properties of the pristine sample and tends even to get slightly more resistive. For a dose rate of $9 \mathrm{mGy} / \mathrm{s}$ and a surface potential of $-300 \mathrm{~V}$,

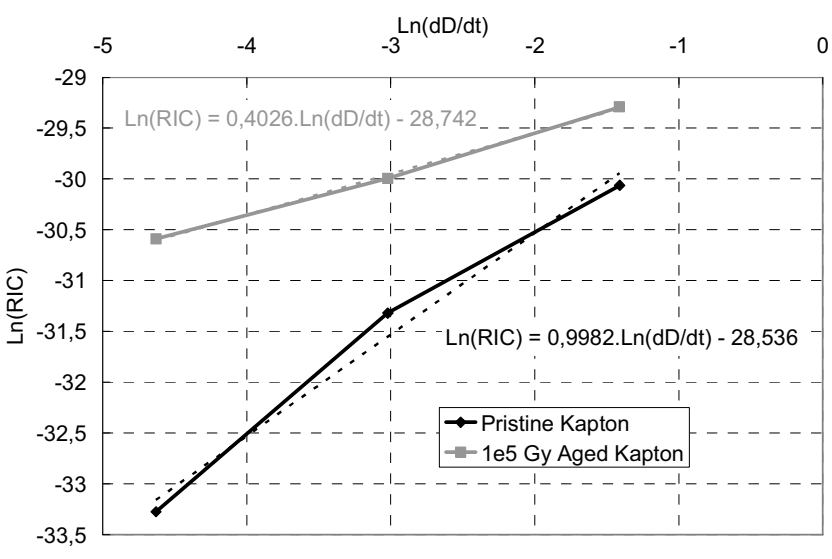

Fig. 9. Evolution of RIC as a function of radiation dose rate for pristine and aged Kapton samples (radiation dose: $10^{5} \mathrm{~Gy}$ ).

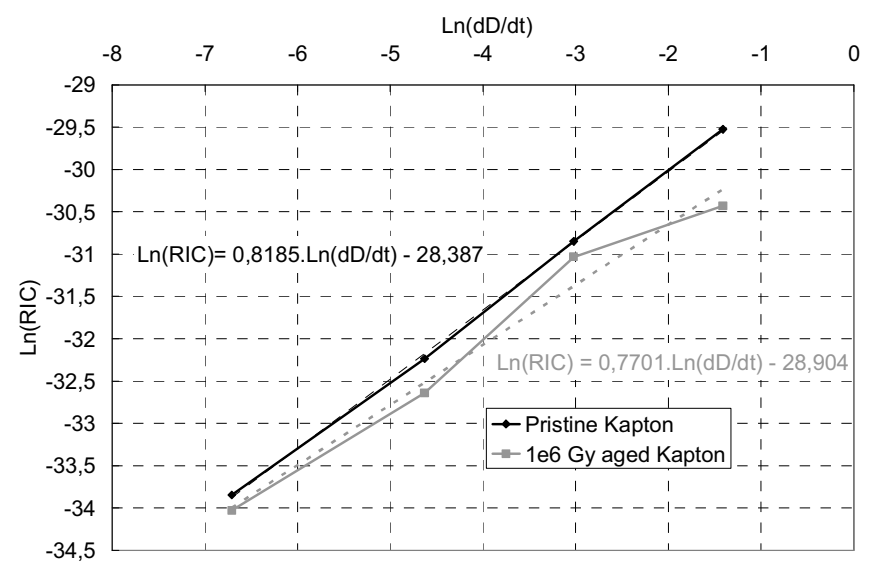

Fig. 10. Evolution of RIC as a function of radiation dose rate for pristine and aged Kapton samples (radiation dose: $10^{6} \mathrm{~Gy}$ ).

radiation-induced conductivity is then equal to $10^{-14} \Omega^{-1} \cdot \mathrm{m}^{-1}$ for the pristine sample versus $7 \times 10^{-15} \Omega^{-1} \cdot \mathrm{m}^{-1}$ for the aged sample. At this radiation dose level, structure alteration induced in Kapton may affect at significant level its electrical properties and aging effect may prevail over the ionization one.

\section{Polyepoxy Adhesive DP490}

The polyepoxy adhesive Scotch-Weld DP490 has been tested following phase 1 described in Section II-B. This material presents specific electric behavior as shown in Fig. 11 that this material does not acquire any significant RIC under high electron energy irradiation. This figure shows the evolution of charging potential for pristine and $10^{6} \mathrm{~Gy}$ aged polyepoxy samples along the different steps described in Section II-B for phase 1 (charging with $20 \mathrm{keV}$, intrinsic relaxation, and enhanced relaxation with $400-\mathrm{keV}$ electron beam). We cannot detect any change in relaxation kinetics when the $400-\mathrm{keV}$ electron beam is switched on for stimulation. This relaxation kinetics is not affected as well by the increase of radiation dose rate. The overall conductivity of pristine DP490 sample keeps up the same level along the different tests, equal to $10^{-14} \Omega^{-1} \cdot \mathrm{m}^{-1}$. Relaxation is therefore not enhanced by the 


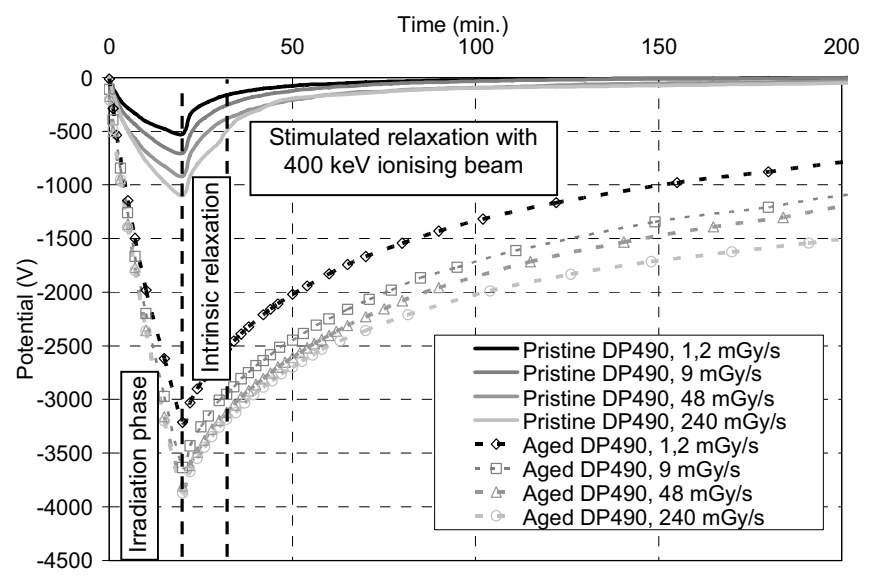

Fig. 11. Evolution of surface potential on pristine and aged polyepoxy DP490 samples (radiation dose : $10^{6} \mathrm{~Gy}$ ) during the different experimental steps for intrinsic and RIC evaluation. Step 1: irradiation with $20 \mathrm{keV}$ electron beam. Step 2: intrinsic relaxation. Step 3: stimulated relaxation with 400-keV electron beam.

400-keV irradiation and its kinetics is not altered by the presence of this irradiation. We can therefore state that ionization process does not affect significantly the overall conductivity of the material at room temperature. For this material, electric conductivity may therefore be preferentially steered by ionic conductivity instead of electron conductivity, which is directly dependent on radiation dose.

The irradiation of the material at a high radiation dose level has, however, an effect on the electrical properties of the polymer as shown in Fig. 11 that pristine material builds up a lower absolute charge surface potential for the same $20-\mathrm{keV}$ irradiation condition (first step of phase 1). From the relaxation kinetics, the conductivity of the aged sample is lower than the pristine one, equal to $2.10^{-15} \Omega^{-1} \cdot \mathrm{m}^{-1}$ (versus $10^{-14} \Omega^{-1} \cdot \mathrm{m}^{-1}$ for polyepoxy pristine samples). As for FEP and Kapton, the polyepoxy DP490 tends therefore to get less conductive when aged with high energy electrons.

To summarize the different experimental analysis on pristine and polyepoxy samples: the $400-\mathrm{keV}$ electron irradiation could then induce ionization of polyepoxy materials but the induced conductivity does not overcome intrinsic bulk conductivity that could be steered by ionic transport. At high dose levels, this high energy radiation can, however, induce physicochemical aging that tends to lower ionic transport and therefore the overall bulk conductivity.

\section{Silicon Adhesive QS1123}

Silicon adhesive QS1123 from MAP has been tested in the same experimental conditions as polyepoxy adhesive DP490. We have been able to notice a slight decrease of the intrinsic conductivity from $3 \cdot 10^{-14} \Omega^{-1} \cdot \mathrm{m}^{-1}$ to $10^{-14} \Omega^{-1} \cdot \mathrm{m}^{-1}$. This feature is conspicuous looking at the fact that the charging potential of the aged sample is higher than for pristine one for the same $20-\mathrm{keV}$ irradiation conditions (Fig. 12). Contrary to DP490, the QS1123 silicon adhesive is submitted to a noticeable RIC that prevails on the intrinsic one for dose rate $>1.2 \mathrm{mGy} / \mathrm{s}$. Electric conductivity is therefore mainly

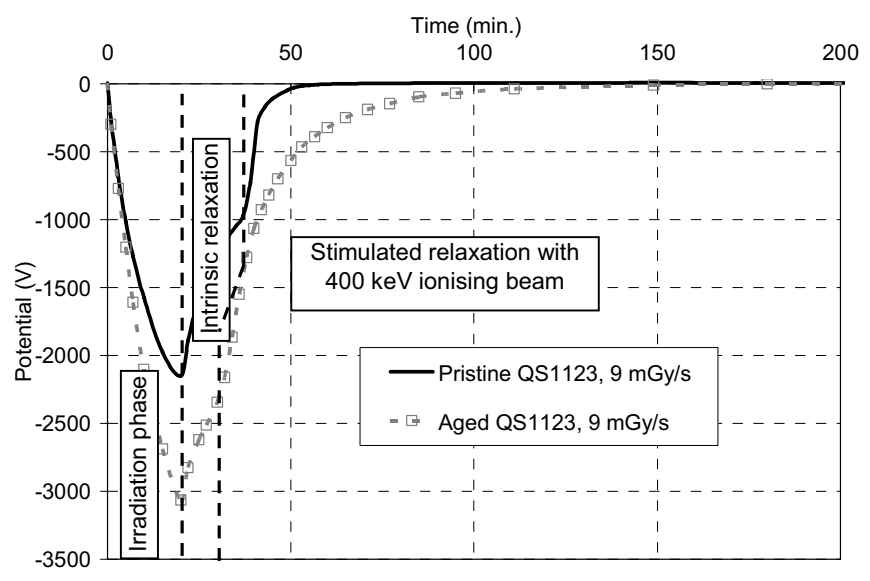

Fig. 12. Evolution of surface potential on pristine and aged silicon QS1123 sample (radiation dose: $10^{6} \mathrm{~Gy}$ ) during the different experimental steps for intrinsic and RIC evaluation. Step 1: irradiation with $20-\mathrm{keV}$ electron beam. Step 2: intrinsic relaxation. Step 3: stimulated relaxation with $400-\mathrm{keV}$ electron beam.

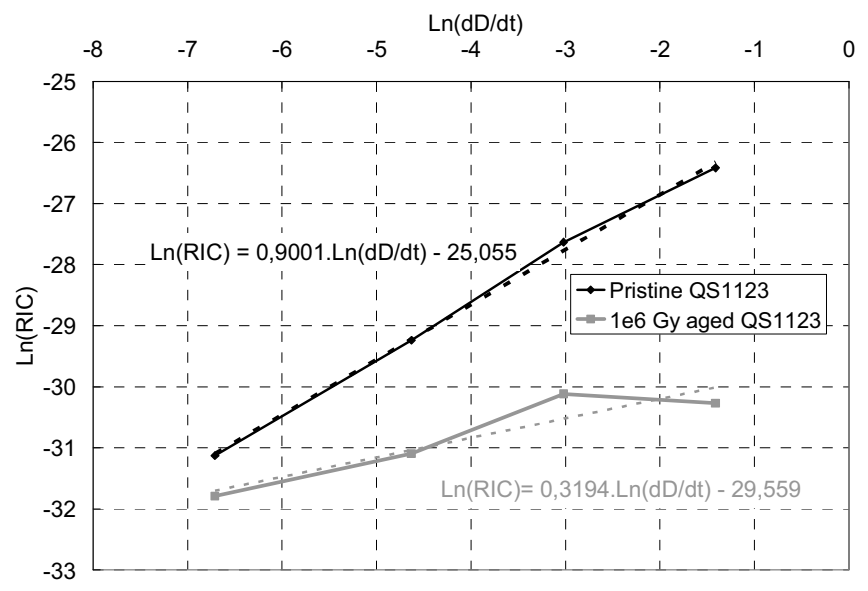

Fig. 13. Evolution of RIC as a function of radiation dose rate for pristine and aged silicon QS1123 samples (radiation dose: $10^{6} \mathrm{~Gy}$ ).

steered by electron mobility in the material. This RIC is, however, noticeably reduced with radiation aging, as shown in Fig. 13.

\section{CONCLUSION}

This paper aimed at studying the effect of high radiation doses induced by high energy electron irradiation over the electric conductivity of space used polymers (Teflon FEP, Kapton, polyepoxy adhesive DP490 and silicon adhesive QS1123). We have been able to demonstrate that radiation aging reveals an overall decrease in intrinsic and RIC (comparison between black and grey curves in $\operatorname{Ln}$ (RIC) charts: grey curves for aged samples are always beneath black curves for pristine samples). A radiation dose threshold might, however, be present for Kapton that is highly conductive for radiation doses $>10^{5}$ Gy. Above this radiation level, structural aging may prevail over ionization leading to a reduction of RIC. It was interesting as well to notice that RIC steers the overall charging process for FEP, Kapton, and silicon QS1123 but this parameter is negligible in comparison with the intrinsic one 
for polyepoxy DP490. The conductivity of this material could therefore be due to ionic conductivity. Finally, we have been able to characterize through successive high energy irradiation steps on Teflon FEP the long-term influence of ionization process on the electrical conductivity that could be reduced because of trapped electron acting as recombination centers.

\section{REFERENCES}

[1] L. Levy, T. Paulmier, B. Dirassen, C. Inguimbert, and M. Van Eesbeek, "Aging and prompt effects on space material properties," IEEE Trans. Plasma Sci., vol. 36, no. 5, pp. 228-2237, Oct. 2008.

[2] T. Paulmier, B. Dirassen, D. Payan, and M. Van Eesbeek, "Material charging in space environment: Experimental test simulation and induced conductive mechanisms," IEEE Trans. Dielectr. Electr. Insul., vol. 16, no. 3, pp. 682-688, Jun. 2009.
[3] B. Gross, "Radiation-induced charge storage and polarization effects," in Electrets, vol. 1, G. M. Sessler, Ed., 3rd ed. Morgan Hill, CA, USA: Laplacian Press, 1998, pp. 217-284.

[4] B. Gross, R. M. Faria, and G. F. Ferreira, "Radiation induced conductivity in Teflon irradiated by X-rays," J. Appl. Phys., vol. 52, no. 2, pp. 571-578, 1981.

[5] R. Hanna, T. Paulmier, P. Molinié, M. Belhaj, B. Dirassen, D. Payan, and N. Balcon, "Radiation induced conductivity under multi-energetic electron beam irradiated Teflon FEP," in Proc. 12th Spacecraft Charging Technol. Conf., May 2012, pp. 10-35.

Authors' photographies and biographies not available at the time of publication. 